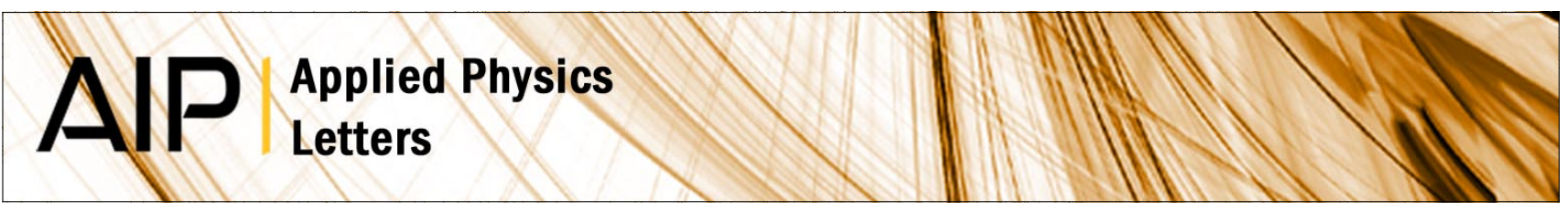

\title{
Electronic structure of delta-doped La:SrTiO3 layers by hard x-ray photoelectron spectroscopy
}

\author{
A. M. Kaiser, A. X. Gray, G. Conti, B. Jalan, A. P. Kajdos et al.
}

Citation: Appl. Phys. Lett. 100, 261603 (2012); doi: 10.1063/1.4731642

View online: http://dx.doi.org/10.1063/1.4731642

View Table of Contents: http://apl.aip.org/resource/1/APPLAB/v100/i26

Published by the American Institute of Physics.

\section{Related Articles}

Bulk and surface half-metallicity: Metastable zinc-blende TiSb

J. Appl. Phys. 112, 023712 (2012)

Electronic structures of the $\mathrm{SrTiO} 3(110)$ surface in different reconstructions

J. Chem. Phys. 137, 044701 (2012)

Electronic structure and thermoelectric properties of nanostructured EuTi1-xNbxO3- $\delta(x=0.00 ; 0.02)$

Appl. Phys. Lett. 101, 033908 (2012)

Negative spin polarization at the Fermi level in Fe4N epitaxial films by spin-resolved photoelectron spectroscopy J. Appl. Phys. 112, 013911 (2012)

Abnormal electronic transition variations of lanthanum-modified lead zironate stannate titanate ceramics near morphotropic phase boundary: A spectroscopic evidence

Appl. Phys. Lett. 101, 011914 (2012)

\section{Additional information on Appl. Phys. Lett.}

Journal Homepage: http://apl.aip.org/

Journal Information: http://apl.aip.org/about/about_the_journal

Top downloads: http://apl.aip.org/features/most_downloaded

Information for Authors: http://apl.aip.org/authors

\section{ADVERTISEMENT}

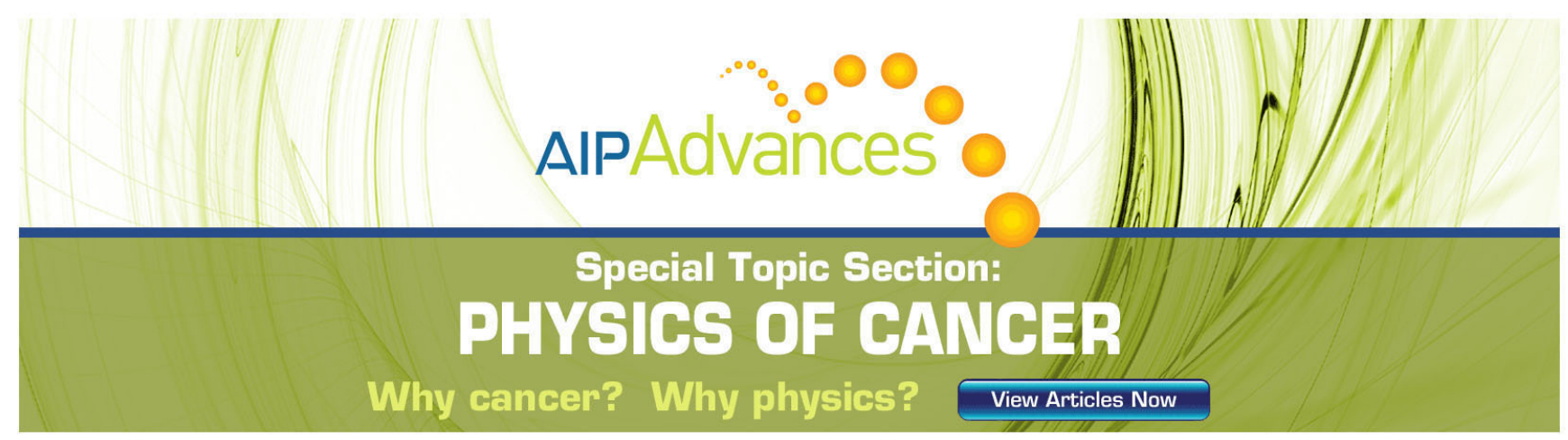




\title{
Electronic structure of delta-doped $\mathrm{La}: \mathrm{SrTiO}_{3}$ layers by hard x-ray photoelectron spectroscopy
}

\author{
A. M. Kaiser, ${ }^{1,2,3}$ A. X. Gray, ${ }^{1,2,4}$ G. Conti, ${ }^{1,2}$ B. Jalan, ${ }^{5,6}$ A. P. Kajdos, ${ }^{5}$ A. Gloskovskii, ${ }^{7}$ \\ S. Ueda, ${ }^{8}$ Y. Yamashita, ${ }^{8}$ K. Kobayashi, ${ }^{8}$ W. Drube, ${ }^{9}$ S. Stemmer, ${ }^{5}$ and C. S. Fadley ${ }^{1,2}$ \\ ${ }^{1}$ Department of Physics, University of California, Davis, California 95616, USA \\ ${ }^{2}$ Materials Sciences Division, Lawrence Berkeley National Laboratory, Berkeley, California 94720, USA \\ ${ }^{3}$ Peter-Grünberg-Institut PGI-6, Forschungszentrum Jülich, 52425 Jülich, Germany \\ ${ }^{4}$ Stanford Institute for Materials and Energy Sciences, SLAC National Accelerator Laboratory, Menlo Park, \\ California 94025, USA \\ ${ }^{5}$ Materials Department, University of California, Santa Barbara, California 93106-5050, USA \\ ${ }^{6}$ Department of Chemical Engineering and Materials Science, University of Minnesota, Twin Cities, \\ Minnesota 55455, USA \\ ${ }^{7}$ Institut für Anorganische Chemie und Analytische Chemie, Johannes Gutenberg-Universität, 55099 Mainz, \\ Germany \\ ${ }^{8}$ NIMS Beamline Station at SPring-8, National Institute for Materials Science, Hyogo 679-5148, Japan \\ ${ }^{9}$ Deutsches Elektronen-Synchrotron (DESY), Notkestrasse 85, 22607 Hamburg, Germany
}

(Received 24 April 2012; accepted 13 June 2012; published online 27 June 2012)

We have employed hard x-ray photoemission (HAXPES) to study a delta-doped $\mathrm{SrTiO}_{3}$ layer that consisted of a 3-nm thickness of $\mathrm{La}$-doped $\mathrm{SrTiO}_{3}$ with $6 \% \mathrm{La}$ embedded in a $\mathrm{SrTiO}_{3}$ film. Results are compared to a thick, uniformily doped $\mathrm{La}_{\mathrm{SrTiO}}$ layer. We find no indication of a band offset for the delta-doped layer, but evidence of the presence of $\mathrm{Ti}^{3+}$ in both the thick sample and the delta-layer, and indications of a density of states increase near the Fermi energy in the delta-doped layer. These results further demonstrate that HAXPES is a powerful tool for the non-destructive investigation of deeply buried doped layers. (C) 2012 American Institute of Physics. [http://dx.doi.org/10.1063/1.4731642]

Recently, it has been shown that high-mobility $\mathrm{SrTiO}_{3}$ (STO) films with excellent control over carrier concentrations can be obtained by La-doping during growth by molecular beam epitaxy (MBE). ${ }^{1}$ Furthermore, it has been shown that by inserting very thin doped layers on the order of a few $\mathrm{nm}$ in thickness between undoped layers (delta-doping), a two-dimensional electron gas is formed. ${ }^{2-4}$ Such delta-doped layers are relevant to understanding two-dimensional quantum phenomena in $\mathrm{SrTiO}_{3},{ }^{5}$ which have attracted attention for their potential of combining unique field-tunable, magnetic and superconducting properties. ${ }^{6-9}$

The presence of a thicker undoped layer above the delta layer, ${ }^{2,3}$ makes studies of the electronic structure of the delta layer by conventional $\mathrm{x}$-ray photoelectron spectroscopy (XPS) difficult, if not impossible, due to the limited electron escape depth. ${ }^{10,11}$ By increasing the photon and thus the electron kinetic energy from the conventional soft $\mathrm{x}$-ray region $(\sim 1 \mathrm{keV})$ into the multi-keV regime, the probing depth, which is dependent on the inelastric mean free path (IMFP) of the photoelectrons, can be significantly increased to several nanometers. ${ }^{12}$ This permits electronic structure studies of such complex oxides that are not limited to the surface region. ${ }^{13,14}$

In this article, we thus use hard X-ray photoelectron spectroscopy (HAXPES) to non-destructively investigate the electronic properties of deeply buried, doped layers, in particular La-doped STO films grown on (001) STO single crystals by MBE. Details regarding growth and the nature of the two-dimensional electron gas in these structures can be be found elsewhere. ${ }^{3}$ The delta-doped structure consists of a 300-nm-thick buffer layer of undoped STO grown on an STO substrate, followed by a 3-nm-thick La-doped STO layer that is finally capped by $4.5 \mathrm{~nm}$ of undoped STO. The La-atoms replace Sr-atoms in the STO unit cells. The relative concentration of $\mathrm{La}$ atoms was set to be $6 \%$, as calibrated by measurements of the charge carrier and $\mathrm{La}$ concentration, and should be accurate to within about $0.2 \%$. ${ }^{1}$ For reference as an effectively "bulk'sample, a 30-nm-thick, uniformly doped film with the composition $\mathrm{La}_{0.06} \mathrm{Sr}_{0.94} \mathrm{TiO}_{3}$ was also grown on STO.

Most of the HAXPES measurements were carried out at the beamline P09 at PETRA III (Hamburg, Germany) with a SPECS PHOIBOS $225 \mathrm{HV}$ hemispherical analyzer. The photon energy was set to $4990 \mathrm{eV}$ and the photon incidence angle was set to $2^{\circ}$, as measured from the sample surface, allowing for near-normal emission of the photoelectrons from the sample and resulting in the highest bulk sensitivity. The experimental resolution using a $\mathrm{Si}(111)$ monochromator was determined to be $600 \mathrm{meV}$ by measurements at the Fermi energy a Au foil sample. The resulting electron IMFP $\lambda$ has been estimated using the TPP- $2 \mathrm{M}$ formula ${ }^{10}$ and is found to be in the range of $6-7 \mathrm{~nm}$. In a recent HAXPES study on similar perovskite materials, however, it has been shown that the effective attenuation length is about $20 \%$ lower than predicted by the TPP-2M formula; ${ }^{15}$ thus, $5-6 \mathrm{~nm}$ is a good estimate of the mean depth of photoelectron emission, and the 30-nm reference film should represent a "bulk" sample as seen by HAXPES. Supplementary measurements were also carried out at beamline BL15XU (Ref. 16) at SPring-8 (Hyōgo, Japan) with the photon energy set to $3237 \mathrm{eV}$, which yields a slightly lower IMFP of about 3$4 \mathrm{~nm}$ via TPP-2M. Using an additional $\mathrm{Si}(220)$ postmonochromator, the experimental resolution at this lower energy case was significantly better at $170 \mathrm{meV}$. The 
measurements at both light sources yielded consistent results. The binding energies shown in the spectra are calibrated by measurements of the Fermi edge of a Au sample. No evidence of sample charging was found in either dataset.

As a comparative comment concerning the advantage of using hard $\mathrm{x}$-ray excitation, the TPP-2M values for IMFPs can be used to estimate the change of intensity $I_{\delta} / I_{b u l k}$, when comparing the photoelectron intensities of the thick La:STO reference film $I_{\text {bulk }}$ to that of the buried delta-layer $I_{\delta}$. For the La $3 d_{5 / 2}$ core levels with a binding energy of $E_{b}=834 \mathrm{eV}$ and a photon energy of $h \nu=4990 \mathrm{eV}$, the resulting IMFP is $\lambda_{\mathrm{La} 3 \mathrm{~d}, 4990 \mathrm{eV}}=6.1 \mathrm{~nm}$, while for the commonly used $\mathrm{Al} \mathrm{K} \alpha$ $\mathrm{x}$-ray source at $1487 \mathrm{eV}$, the resulting IMFP is only $\lambda_{\mathrm{La} 3 \mathrm{~d}, 1486 \mathrm{eV}}=1.4 \mathrm{~nm}$. Using the formula below and integrating over depth $\mathrm{z}$,

$$
\frac{I_{\delta}}{I_{\text {bulk }}}=\frac{\int_{z=4.5 \mathrm{~nm}}^{7.5 \mathrm{~nm}} e^{-z / \lambda} \mathrm{dz}}{\int_{z=0}^{\infty} e^{-z / \lambda} \mathrm{dz}},
$$

we can calculate the fraction of the intensities of the delta-layer and the bulk La:STO reference film for different energies. For simplicity in the calculation, the reference layer has been assumed to have an infinite thickness, which changes the final results by less than $1 \%$. We then obtain $I_{\delta} / I_{\text {bulk }}=0.185$ for a photon energy of $4990 \mathrm{eV}$ and $I_{\delta} / I_{\text {bulk }}=0.035$ for $\mathrm{Al} \mathrm{K} \alpha$ radiation. The intensity of the buried film is thus significantly increased by a factor of 5 due to the higher photon energy. The 0.185 ratio at the energy of our measurements has also been confirmed using more quantitative calculations based on the SESSA program, which includes the effects of elastic scattering, and yields a value of 0.189 very close to it. ${ }^{17}$

Broad-range survey spectra collected from both samples are shown in Fig. 1. We observe all expected features from the elements Sr, Ti, O, and La from the STO and La:STO layers, respectively, as well as $\mathrm{C}$ from a few- $\AA$-thick layer of carbon- and probably also oxygen-containing adsorbates on the surface. ${ }^{18}$ Closer inspection of the data shows a small amount of $\mathrm{Cu}$ (e.g., with $2 p$ binding energies at $\sim 933$ and $952 \mathrm{eV}$, and too weak to see in Fig. 1) that is attributed to surface contaminations due to contact with $\mathrm{Cu}$ parts after the deposition. By comparison of the relative peak areas with simulations using the SESSA package, ${ }^{17}$ the $\mathrm{Cu}$ amount in the investigated area could be quantified to be only 0.05 monolayers on the sample surface, and thus it can be neglected in discussing our core-level spectra and the critical valence-band region near the Fermi level, which is in turn expected based on photoelectric cross sections at this energy to be dominated by $\mathrm{Ti} 4 s$ character. Secondary ion mass spectrometry (SIMS) measurements performed immediately after deposition on similar samples showed no evidence of $\mathrm{Cu}$ in the film, suggesting that some $\mathrm{Cu}$ contamination occurred either during transport measurements or during mounting the sample on a $\mathrm{Cu}$ sample holder.

Figure 2 shows the spectra acquired for the La $3 d$, Ti $2 p$, Sr $3 p$ core levels, and for the valence-band region. Due to the low La concentration and the capping layer for the deltadoped sample, the signal-to-background ratio at the peak

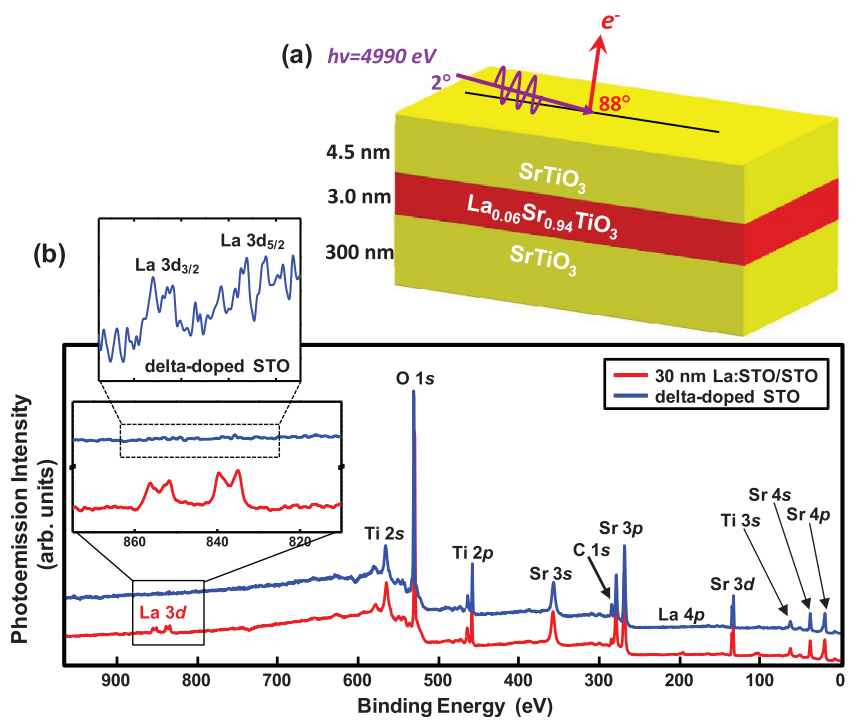

FIG. 1. (a) Experimental geometry, including sample configuration with a $\mathrm{La}_{0.06} \mathrm{Sr}_{0.94} \mathrm{TiO}_{3}$ doped delta layer embedded in undoped $\mathrm{SrTiO}_{3}$. (b) Survey spectra obtained with a photon energy of $4990 \mathrm{eV}$ and covering a range of $0-1000 \mathrm{eV}$ binding energy from a thick $30 \mathrm{~nm}$ La-doped STO film (red curve) and from the delta-doped layer of (a) with the same La concentration (blue curve). Various peaks are labeled. The spectra have been offset vertically for clarity. A blowup of the $\mathrm{La} 3 d$ region for both samples is also shown.

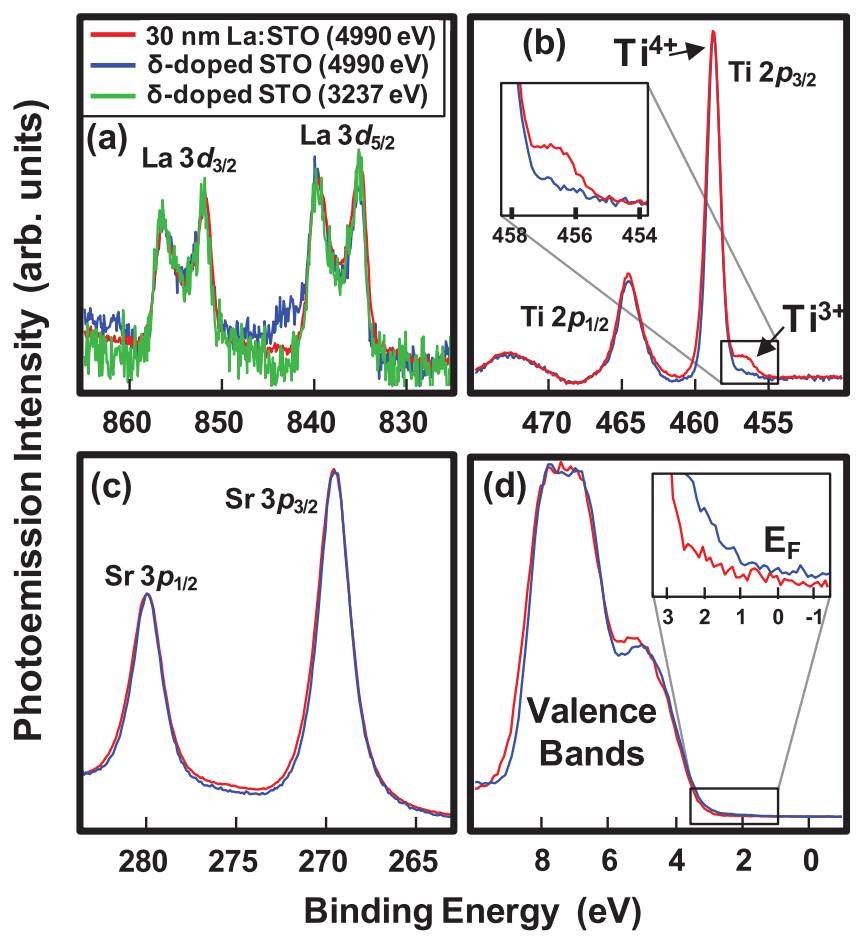

FIG. 2. Core level and valence band spectra of 30-nm-thick La:STO and the delta-doped layer of Fig. 1(a). (a) La 3d, obtained at $4990 \mathrm{eV}$ for the thick sample, and at both 4990 and $3237 \mathrm{eV}$ for the delta-doped layer. Spectra obtained with $4990 \mathrm{eV}$ for (b) Ti $2 p$, with the inset showing the low-bindingenergy $\mathrm{Ti}^{3+}$ features, (c) $\mathrm{Sr} 3 p$, and (d) the valence band region. All spectra are normalized to the peak maxima. The La $3 d$ spectra of the delta-doped sample have been generated by subtracting a linear background from the measured spectra. The small differences between the blue spectra in (a) and the other two were found to be a short-term beam instability that led to experimental artifacts in intensity. None of our other data was affected by this problem. 
maximum for $\mathrm{La} 3 d$ is only $2 \%$. Therefore, the spectra in Fig. 2(a) have been plotted after the subtraction of a linear background. Remarkably, after the background subtraction and normalization to a uniform height, the doubling of each of the La $3 d 3 / 2$ and $5 / 2$ peaks that is attributed to final-state screening satellites ${ }^{19}$ is clearly visible, even for the deltadoped sample. The positions of all peaks, the splittings of the different components, and their spectral weights agree excellently for spectra obtained from the reference layer and at two different photon energies. As the splitting and the spectral distribution of the components for each core level ( $\mathrm{La}$ $3 d_{3 / 2}$ and La $3 d_{1 / 2}$ ) strongly depends on the interaction between the La-atoms and their surrounding atoms, ${ }^{20}$ we conclude that for both samples (delta-doped layer and bulk), at least $90 \%$ of the La atoms are in the same chemical state and environment, thus confirming the quality of the samples. As a further analysis step, we have compared the La $3 d_{5 / 2}$ core level intensities to the intensities of the Ti $2 p_{3 / 2}$ peaks representing atoms present in both samples with the same concentration, and determined the ratio $R=I_{L_{\text {La3d }} / 2} /$ $I_{T i 2 p_{3 / 2}}$. The resulting experimental value of this ratio $\frac{R_{\delta \text {-layer }}}{R_{\text {bulk }}}=$

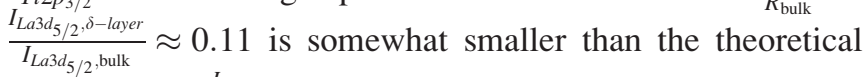
prediction of $\frac{I_{\delta}}{I_{\text {bulk }}} \approx 0.187$, but this discrepancy can easily be attributed to a too high IMFP in STO as obtained from the TPP-2M formula, small inaccuracies in the determination of the true peak intensities and/or the delta-layer and capping layer thicknesses.

Having confirmed the chemical environment of the La ions, it is instructive to analyze spectra of the other core levels. Due to the thick STO capping layer of the delta-doped sample, the spectra of Sr and Ti core levels of this sample are strongly dominated by the undoped capping layer. Comparing spectra of this sample to the La:STO sample could thus give hints to the presence of any interface charging and/ or band offsets between the capping layer and the deltalayer. ${ }^{21}$ Comparison of the positions of the main features in all spectra, however, yields no measurable shifts. Thus, it can be concluded that no significant band offsets are present at the interface between the capping layer of STO and the delta-doped layer. This is also confirmed by the fact that the La $3 d$ spectra do not show any significant broadening for the delta-layer, as would be observed if the electronic bands are bent over a length comparable to the IMFP. ${ }^{22}$

In contrast to the delta-doped sample, Fig. 2(b) shows that, for the thick La:STO film, two peaks are present for the Ti $2 p_{3 / 2}$ component, and these we assign to the presence of both $\mathrm{Ti}^{4+}$ and $\mathrm{Ti}^{3+}$ as indicated. In fact, fitting the two components for the thick sample yields a spectral weight of about $8.5 \pm 2.3 \%$ of the lower-binding feature assigned to $\mathrm{Ti}^{3+}$. This value is in good agreement with the assumed Laconcentration of $6 \%$ and thus supports the assumption that on average for every La-atom on a $\mathrm{Sr}$-site, one Ti-atom changes its charge state from $\mathrm{Ti}^{3+}$ (that of $\mathrm{SrTiO}_{3}$ ) to $\mathrm{Ti}^{4+}$ (that of $\mathrm{LaTiO}_{3}$ ). ${ }^{23}$ Although the Ti $2 p_{1 / 2}$ peak for the thick sample does not show as obviously a second component, this is consistent with prior analyses where the occurrence of different Ti charge states only led to a small asymmetry of the Ti $2 p_{1 / 2}$ peak, probably due to energy broadening associated with the shorter lifetime through Coster-Kronig decay $\left(L_{2} L_{3} V\right) .{ }^{24}$ Looking carefully at the delta-doped sample (see inset in Fig. 2(b)), we can also see evidence of $\mathrm{Ti}^{3+}$ as a sloping shoulder. Fitting this spectrum with the same peak parameters as those describing the thick La:STO film yields a ratio of $0.03 \pm 0.01$ that is very close to an estimated value from the bulk sample of 0.085 times the theoretical average ratio of $0.187=0.016$, thus providing an additional selfconsistency check of our results.

Figure 2(c) now compares the Sr $3 p$ peaks of both samples. Here, no major changes are expected, as even for films where a small number of $\mathrm{Sr}$ atoms is replaced by La atoms, the remaining $\mathrm{Sr}$ atoms are still in the same chemical environment as $\mathrm{Sr}$ in pure $\mathrm{SrTiO}_{3}$. This is confirmed by our measurements, in which no significant differences are observed.

Figure 2(d) finally shows the valence band region. For both films we observe a non-zero density of states (DOS) up to the Fermi level, which is supported by the electrical measurements showing metallic behavior for both systems, ${ }^{1,3}$ although as might be expected, the DOS near $\mathrm{E}_{\mathrm{F}}$ is weaker due to the lower number of $\mathrm{La}$ atoms contributing.

Our results for La:STO can also be directly compared to a recent HAXPES study of the $2 \mathrm{D}$ electron gas at the interfaces in $\mathrm{LaAlO}_{3}-\mathrm{SrTiO}_{3}$ heterostructures. ${ }^{25}$ In this work, the same feature in the Ti $2 p_{3 / 2}$ spectrum that is linked to $\mathrm{Ti}^{3+}$ is observed, and its analysis as a function of electron exit angle, and thus varying interface sensitivity, permitted estimating the thickness of the $2 \mathrm{D}$ electron gas at the interface. What we see here is a complementary result in that the La doping induces the presencs of $\mathrm{Ti}^{3+}$ in both the thick sample and the delta layer, although as expected, much more weakly seen in the latter. By coincidence, the relative intensity of the $\mathrm{Ti}^{3+}$ in our thick sample and in the most interface sensitive spectrum of this prior study (Fig. 1(a) of Ref. 25 are almost identical).

In summary we have conducted a HAXPES study on a La-doped STO film and delta-layer with the same Laconcentration. Although the delta-layer was capped by a $4.5 \mathrm{~nm}$ thick layer that would not permit studies of the electronic structure by soft x-ray photoelectron spectroscopy, we have been able to collect spectra of the La $3 d$ core levels of the delta-layer. Comparing these results to spectra collected with a thick "bulk" reference sample, we see no significant differences, leading to the conclusion that $\mathrm{La}$ atoms have the same chemical environment in both layers. Comparing the energy spacing of the La $3 d$ peaks relative to other core levels also showed no evidence of shifts due to charging or valence band shifts or offsets. Two peaks present in the Ti $2 p$ spectra of the reference La:STO sample further indicate the presence of different charge states of the Ti atoms depending on the bonding to $\mathrm{Sr}$ or La atoms; there is also evidence of these two charge states in the delta-doped layer, although it is not possible to as cleanly resolve it due to the overwhelming signal of the undoped STO. These results thus clarify the electronic structure in an important delta doped system and further demonstrate the power of HAXPES for electronic structure investigations of buried materials even for very low doping concentrations.

Travel and salary support for A.X.G. and A.K. was provided by the MURI program of the Army Research Office 
(Grant No. W911-NF-09-1-0398). Two of us (C.S.F. and G.C) also acknowledge salary support from the U.S. Department of Energy under Contract No. DE-AC02-05CH11231. Research at Stanford was supported through the Stanford Institute for Materials and Energy Science (SIMES) and the LCLS by the US Department of Energy, Office of Basic Energy Sciences. The HAXPES measuements at BL15XU of SPring-8 were performed under the approval of NIMS Beamline Station (Proposal No. 2010B4800). The authors are grateful to HiSOR, Hiroshima University and JAEA/ SPring-8 for the development of the HAXPES station at BL15XU of SPring-8. S.S. also acknowledges funding from NSF (Grant No. DMR-1006640). The HAXPES instrument at beamline P09 is jointly operated by the University of Würzburg (R. Claessen), the University of Mainz (C. Felser) and DESY. Funding by the Federal Ministry of Education and Research (BMBF) under contracts 05KS7UM1, 05K10UMA, 05KS7WW3, and 05K10WW1 is gratefully acknowledged.

${ }^{1}$ J. Son, P. Moetakef, B. Jalan, O. Bierwagen, N. J. Wright, R. EngelHerbert, and S. Stemmer, Nat. Mater. 9, 482 (2010).

${ }^{2}$ Y. Kozuka, M. Kim, C. Bell, B. G. Kim, Y. Hikita, and H. Y. Hwang, Nature (London) 462, 487 (2009).

${ }^{3}$ B. Jalan, S. Stemmer, S. Mack, and S. J. Allen, Phys. Rev. B 82, 081103 (2010).

${ }^{4}$ Y. Kozuka, M. Kim, H. Ohta, Y. Hikita, C. Bell, and H. Y. Hwang, Appl. Phys. Lett. 97, 222115 (2010).

${ }^{5}$ P. V. Ong, J. Lee, and W. E. Pickett, Phys. Rev. B 83, 193106 (2011).

${ }^{6}$ J. Mannhart, D. H. A. Blank, H. Y. Hwang, A. J. Millis, and J. M. Triscone, MRS Bull. 33, 1027 (2008).

${ }^{7}$ A. D. Caviglia, M. Gabay, S. Gariglio, N. Reyren, C. Cancellieri, and J. M. Triscone, Phys. Rev. Lett. 104, 126803 (2010).
${ }^{8}$ M. Ben Shalom, M. Sachs, D. Rakhmilevitch, A. Palevski, and Y. Dagan, Phys. Rev. Lett. 104, 126802 (2010).

${ }^{9}$ K. Ueno, S. Nakamura, H. Shimotani, A. Ohtomo, N. Kimura, T. Nojima, H. Aoki, Y. Iwasa, and M. Kawasaki, Nat. Mater. 7, 855 (2008).

${ }^{10}$ S. Tanuma, C. J. Powell, and D. R. Penn, Surf. Int. Anal. 21, 165 (1993).

${ }^{11}$ S. Tanuma, C. J. Powell, and D. R. Penn, Surf. Int. Anal. 43, 689 (2011).

${ }^{12}$ K. Kobayashi, Nucl. Instrum. Methods Phys. Res. A 601, 32 (2009).

${ }^{13}$ H. Tanaka, Y. Takata, K. Horiba, M. Taguchi, A. Chainani, S. Shin, D. Miwa, K. Tamasaku, Y. Nishino, T. Ishikawa, E. Ikenaga, M. Awaji, A. Takeuchi, T. Kawai, and K. Kobayashi, Phys. Rev. B 73, 094403 (2006).

${ }^{14}$ F. Offi, N. Mannella, T. Pardini, G. Panaccione, A. Fondacaro, P. Torelli, M. W. West, J. F. Mitchell, and C. S. Fadley, Phys. Rev. B 77, 174422 (2008).

${ }^{15}$ A. X. Gray, A. Janotti, J. Son, J. M. LeBeau, S. Ueda, Y. Yamashita, K. Kobayashi, A. M. Kaiser, R. Sutarto, H. Wadati, G. A. Sawatzky, C. G. Van de Walle, S. Stemmer, and C. S. Fadley, Phys. Rev. B 84, 075104 (2011).

${ }^{16}$ S. Ueda, Y. Katsuya, M. Tanaka, H. Yoshikawa, Y. Yamashita, S. Ishimaru, Y. Matsushita, and K. Kobayashi, AIP Conf. Proc. 1234, 403 (2010).

${ }^{17}$ W. Smekal, W. S. M. Werner, and C. J. Powell, Surf. Int. Anal. 37, 1059 (2005).

${ }^{18}$ B. Jalan, J. Cagnon, T. E. Mates, and S. Stemmer, J. Vac. Sci. Technol. A 27, 1365-1368 (2009).

${ }^{19}$ A. Kotani, M. Okada, T. Jo, A. Bianconi, A. Marcelli, and J. C. Parlebas, J. Phys. Soc. Jpn. 56, 798 (1987).

${ }^{20}$ D. J. Lam, B. W. Veal, and D. E. Ellis, Phys. Rev. B 22, 5730 (1980).

${ }^{21}$ S. A. Chambers, Y. Liang, Z. Yu, R. Droopad, J. Ramdani, and K. Eisenbeiser, Appl. Phys. Lett. 77, 1662 (2000).

${ }^{22}$ H. Tanaka, I. Satoh, T. Kanki, E. Ikenaga, M. Kobata, J. J. Kim, S. Ueda, and K. Kobayashi, Appl. Phys. Lett. 98, 133505 (2011).

${ }^{23}$ M. S. J. Marshall, D. T. Newell, D. J. Payne, R. G. Egdell, and M. R. Castell, Phys. Rev. B 83, 035410 (2011).

${ }^{24}$ S. Hashimoto and A. Tanaka, Surf. Int. Anal. 34, 262 (2002).

${ }^{25}$ M. Sing, G. Berner, K. Goß, A. Müller, A. Ruff, A. Wetscherek, S. Thiel, J. Mannhart, S. A. Pauli, C. W. Schneider, P. R. Willmott, M. Gorgoi, F. Schäfers, and R. Claessen, Phys. Rev. Lett. 102, 176805 (2009). 